\title{
Antidepressants in bipolar depression: yes, no, maybe?
}

\author{
Gin S Malhi ${ }^{1,2,3}$
}

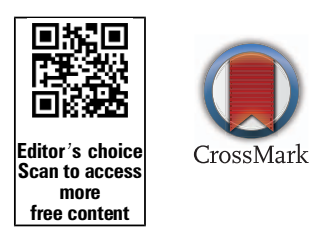

${ }^{1}$ Academic Department of Psychiatry, Kolling Institute, St Leonards, New South Wales, Australia; ${ }^{2}$ Sydney Medical School-Northern, The University of Sydney, St Leonards, New South Wales, Australia; ${ }^{3}$ Department of Psychiatry, CADE Clinic, Royal North Shore Hospital, Northern Sydney Local Health District, St Leonards, New South Wales, Australia

Correspondence to Professor Gin S Malhi, CADE Clinic, Department of Psychiatry, Royal North Shore Hospital, Northern Sydney Local Health District, Level 3, Main Hospital Building, St Leonards, NSW 2065, Australia; gin.malhi@sydney.edu.au

\section{ABSTRACT}

Antidepressants are widely used in the treatment of bipolar depression despite relatively meagre evidence for their efficacy and significant concerns that their prescription can precipitate an acute affective switch into mania/hypomania and that long-term administration can lead to mood instability. Therefore, the use of antidepressants to treat bipolar depression is an important but contentious issue that two recent studies, which provide important new evidence, attempt to inform. One study suggests that long-term continuation of antidepressants in patients with rapid-cycling bipolar disorder leads to a threefold increase in mood episodes during the first year of follow-up - supporting the notion that antidepressants can cause more harm than good, and that they should be used sparingly. However, this is countered by findings from the other study, which suggests that continuation antidepressant monotherapy provides patients with bipolar II disorder reasonable prophylaxis, and that the risk of switching into mania/ hypomania is actually quite low. In addition to contrary findings both studies are modest in sample sizes and have significant design limitations and hence the debate remains unresolved. This brief perspective presents both views in the context of evidence and provides some key insights into the complexity of this challenging but common clinical issue.

\section{INTRODUCTION}

The use of antidepressants to treat bipolar depression is inevitable. First because the natural history of the illness is such that for the majority of patients their initial presentation is in the depressive phase and therefore they are assigned a diagnosis of major depression for which they are treated accordingly, until a manic/hypomanic episode eventuates. Second the only pharmacotherapy alternatives are antipsychotics, anticonvulsants or lithium - none of which (like antidepressants) were developed specifically to treat bipolar depression. Finally, in many instances antidepressants are found to be effective and of sufficient benefit to warrant a trial.

However, in practice the problem antidepressants face is that they are not seemingly as effective in treating bipolar depression as they are in treating major depression and the outcome is largely unpredictable. Furthermore, in some instances administration even at therapeutic dosage can cause a treatment-emergent affective switch (TEAS; see figure 1) and precipitate mood instability (cycling). Thus most guidelines advocate the use of antidepressants as adjuncts to mood stabilising medications. Even recent meta-analyses provide inconsistent findings regarding the efficacy of antidepressants in bipolar depression. ${ }^{1}{ }_{2}$ Hence in practice, some patients benefit from the judicious use of antidepressants whereas others fail to respond and in addition run the risk of mood destabilisation.

Consequently, the use of antidepressants in the treatment of bipolar depression remains controversial and benefits versus potential risks remain unknown. This uncertainty about a key treatment modality prompted a body of experts to form a task force under the auspices of the International Society for Bipolar Disorders (ISBD) and develop a report that addresses the use of antidepressants in bipolar depression. ${ }^{3}$ This ISBD task Force comprising 67 experts from around the world used the Delphi method to develop consensus iteratively. The report acknowledged the widespread use of antidepressants in the treatment of bipolar depression but at the same noted the weak evidence base for their efficacy and safety. A paucity of data limited the recommendations of the task force, which noted that some patients with bipolar may benefit from antidepressants and that regarding side effects selective serotonin reuptake inhibitors were less likely to precipitate switching than tricyclic antidepressants and dual action agents. Furthermore, switching into mania was thought to be more likely in patients with bipolar I disorder, relative to those with bipolar II disorder and that, where possible, antidepressant use should be adjunctive to mood stabilisers as opposed to monotherapy.

Among the 12 recommendations made by the task force regarding antidepressant use in bipolar depression two that are particularly interesting, because of their direct clinical relevance, are worthy of further consideration. First, is the suggestion that antidepressants should be discouraged when there is a history of emergent mania /hypomania or mixed episodes in the context of antidepressant treatment, and that antidepressant use should be avoided in patients with bipolar who have a high level of mood instability or a history of rapid cycling. In contrast, the second recommendation is that maintenance treatment with adjunctive antidepressant medication should be considered an option if a patient relapses into a depressive episode when antidepressant therapy is stopped. Two recent publications both appearing this year in the Journal of Affective Disorders explore these issues further.

\section{YES: ANTIDEPRESSANTS PREVENT SLIDING INTO DEPRESSION}

The first study, conducted by Amsterdam et al, ${ }^{4}$ suggests that subsyndromal depression is more likely in bipolar disorder if antidepressants are not maintained. This study examined the relative long-term efficacy of antidepressant versus mood stabiliser monotherapy in bipolar II disorder. It is the first double-blind randomised, parallel group 6-month relapse prevention study examining the safety and effectiveness of continuation antidepressant versus mood stabiliser monotherapy in bipolar II disorder subjects recovered from acute depression after 12 weeks. The execution of this study in patients with bipolar II disorder is important because they predominantly experience depressive episodes and generally practice guidelines for long-term therapy recommend mood stabiliser monotherapy and the discontinuation of antidepressants after recovery-usually within 3-5 months.

This study randomised 129 depressed patients to treatment with venlafaxine or lithium and found that venlafaxine was more effective than lithium during acute monotherapy with double the number of subjects responding (44 vs 22). During acute treatment and subsequent continuation treatment these numbers dropped to 40 and 15 , respectively, and though the main outcome was depressive relapse, the study also noted the occurrence of treatment-emergent hypomanic symptoms. The study found that the two medications were similar in preventing depressive relapse and had no significant differences in the rate of treatment 


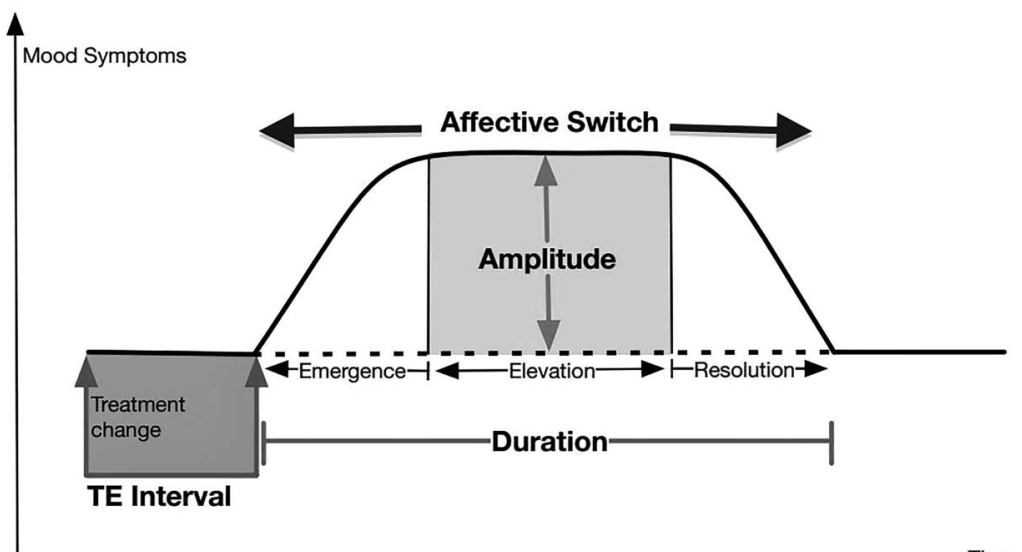

Time

Figure 1 A treatment-emergent affective switch (TEAS) into mania. A TEAS can culminate in either mania/hypomania or depression. The figure shows a switch from euthymia or a depressed state into a state of hypomania/mania (note the duration and number of manic symptoms may not satisfy criteria for an episode of hypomania/mania - and it may be that the switch is marked solely by a few symptoms of mania lasting a relatively short period of time). The diagram also shows the key components of the process that leads to the development of TEAS beginning with a significant change to treatment some time prior to the emergence of mood symptoms. This period of time, called the Treatment-Emergence (TE) interval, is typically of 812 weeks duration according to an International Society for Bipolar Disorders (ISBD) taskforce. However, in practice much shorter intervals are common. The direction of the switch, the amplitude of the change and its overall duration define TEAS. The latter can be subdivided into three components: emergence, elevation and resolution. Adopting a more sophisticated approach to defining TEAS should facilitate a clearer and deeper understanding of this phenomenon to emerge. (Adapted from Malhi et al; ${ }^{10}$ original diagram developed with assistance from Danielle Gessler).

emergent hypomanic symptoms. However interestingly, subsyndromal depressive episodes during continuation monotherapy were greater in lithium-treated subjects as compared to those prescribed venlafaxine. It is important to note that the lithium sample size during continuation therapy was small, and perhaps treating everyone with venlafaxine and then randomising responders to either lithium or venlafaxine could have achieved a larger number of responders. However this would have been problematic for different reasons because of withdrawal from venlafaxine in order to switch to lithium.

The findings of this study are in keeping with prior research and suggest that continuation antidepressant monotherapy of bipolar II disorder confers reasonable prophylaxis with a low risk of switching into mania/hypomania. This is counter to the recommendations of many practice guidelines for long-term maintenance therapy for bipolar II disorder, which instead generally recommend mood stabiliser monotherapy and the discontinuation of antidepressant medication within 1220 weeks after recovery. Of note, this timeframe is shorter than that advocated for unipolar major depression and the reason for this desire to curtail prophylactic antidepressant therapy in the treatment of bipolar depression is the underlying concern that this is likely to induce mania.

\section{NO: ANTIDEPRESSANTS RISK CYCLING OUT OF CONTROL}

The second study, conducted by El-Mallakh et a/ ${ }^{5}$ tackled the difficult issue of antidepressant use in rapid cycling bipolar disorder and found that long-term continuation of antidepressants in patients with rapidcycling bipolar disorder was associated with a threefold increase in mood episodes during the first year of follow-up. It is an important study because it is the first such study to examine the effects of modern antidepressants in the context of a randomised clinical trial. Nested within the Systematic Treatment Enhancement Programme for bipolar disorder (STEP-BD) the researchers investigated those patients with bipolar who initially responded to antidepressant treatment in conjunction with a mood stabiliser.

Specifically, all 68 patients with bipolar achieved and maintained euthymia for at least 2 months and were then randomised to continue antidepressant therapy or discontinue antidepressant treatment while continuing on a mood stabiliser. The number of patients that met criteria for rapid cycling was small $(n=16)$ but most patients in this group experienced 2-4 depressive episodes during follow-up as compared to non-rapid cyclers who typically had few, if any, depressive episodes. Interestingly, in patients with rapid cycling bipolar disorder the long-term continuation of antidepressants resulted in a threefold increase in the rate of depressive episodes in the first year of follow-up. It is important to note that this is distinct from switching into mania or hypomania from depression and this is significant, as pointed out by the authors, because it suggests this is a separate process from rapid cycling per se. Also of significance is the fact that patients were taking mood stabilisers at baseline and that these were not protective with respect to worsening of depressive symptoms and the development of depression.

\section{MAYBE: WHAT TO DO IN PRACTICE?}

Clinically, antidepressants remain a common option for the treatment of bipolar depression, but should they be prescribed and, if so, when?

The extant literature suggests that antidepressants should generally be avoided when treating patients with bipolar depression who are at particular risk of TEAS, have depression with mixed features, rapid cycling or have had a poor response to antidepressants in the past. ${ }^{3}$ However, in individuals with bipolar depression who do not have these features/ risk factors antidepressants may be of some benefit and their use should be considered on an individual basis so as to prevent, in particular, the recurrence of depression. Furthermore, where possible, antidepressant monotherapy should be avoided and antidepressants should be used adjunctively alongside mood stabilising medications-especially those with putative antimanic properties.

\section{CONCLUSION}

Based on how much we know - the only clear inference that can be drawn is that we need to know much more. In order to achieve this however, bipolar depression needs to be better defined and hypothesisdriven randomised controlled trials need to be conducted to better establish the effects of antidepressant therapy.

A key stumbling block thus far, has been the imprecision with which bipolar disorder is diagnosed. For instance, antidepressants are thought to be more useful in bipolar II disorder but both the upper and lower boundaries of this subtype are difficult to define. ${ }^{6}$ Recent developments in the definition of mixed states in Diagnostic and Statistical Manual Fifth Edition 
employing the use of 'mixed features specifiers' has meant that distinguishing bipolar and unipolar depression has become more difficult. ${ }^{7} 8$ Similarly, identifying and describing subsyndromal depressive states is problematic. These problems of definition make it difficult to identify specific start and end points for treatment trials. Consequently, some years ago another ISBD task force standardised bipolar disorder nomenclature ${ }^{9}$ that can be used for treatment trials in bipolar depression.

Further studies focusing on the specific use of antidepressants in bipolar depression are urgently needed. Ideally these studies should be randomised, prospective trials that target both the benefits and risks of antidepressant use. In addition given the burden of bipolar depression, perhaps it is time new molecules with antidepressant properties are developed with a view to not only achieve remission and maintain wellness but actively prevent recurrence. Until such a time, when there is a deeper understanding of bipolar depression and its treatment, its clinical management will remain a challenge and periodically necessitate the sophisticated use of antidepressants.

Competing interests GSM received research funding from AstraZeneca, Eli Lilly, Organon, Pfi zer, Servier and Wyeth; GSM is a speaker for AstraZeneca, Eli Lilly, Janssen-Cilag, Lundbeck, Pfi zer, Ranbaxy, Servier and Wyeth; and a consultant for AstraZeneca, Eli Lilly, Janssen-Cilag, Lundbeck and Servier.

doi:10.1136/eb-2015-102229

Accepted 16 September 2015

\section{REFERENCES}

1. Sidor MM, MacQueen GM. antidepressants for the acute treatment of bipolar depression: a systematic review and meta-analysis. J Clin Psychiatry 2011;72:156-67.

2. Vazquez G, Tondo L, Baldessarini R. Comparison of antidepressant responses in patients with bipolar versus unipolar depression: a meta-analytic review. Phamracopsychiatry 2011;44:21-6.

3. Pacchiarotti I, Bond DJ, Baldessarini RJ, et al. The International Society for Bipolar Disorders (ISBD) task force report on antidepressant use in bipolar disorders. Am J Psychiatry 2013;170:1249-62.

4. Amsterdam JD, Lorenzo-Luaces L, Soeller I, et al. Safety and effectiveness of continuation antidepressant versus mood stabilizer monotherapy for relapse-prevention of bipolar II depression: a randomized, double-blind, parallel-group prospective study. J Affect Disord 2015;185:31-7.

5. El-Mallakh RS, Vöhringer PA, Ostacher MM, et al. Antidepressants worsen rapid-cycling course in bipolar depression: a STEP-BD randomized clinical trial. J Affect Disord 2015;184:318-21.

6. Malhi G, Berk M. Diagnosing bipolar disorder: defining thresholds and setting boundaries. Aust N Z J Psychiatry 2014;48:500-4.

7. Malhi GS. Diagnosis of bipolar disorder: who is in a mixed state? Lancet 2013;381:1599-600.

8. American Psychiatric Association. Diagnostic and statistical manual of mental disorders. 5th Edn. American Psychiatric Association, 2013.

9. Tohen M, Frank E, Bowden CL, et al. The International Society for Bipolar Disorders (ISBD) Task Force report on the nomenclature of course and outcome in bipolar disorders. Bipolar Disord 2009:11:453-73.

10. Malhi GS, Masson M, Bellivier F. Teasing apart Bipolar III: The causes and consequences of a Treatment-Emergent Affective Switch (TEAS) into mania. Aust N Z J Psychiatry 2015;49:866-8. 